\title{
Processing and Characterization of Silicon Nitride Nanofiber Paper
}

\author{
Kei-Peng Jen, Ronald Warzoha, Ji Guo, Michael Tang, and Sridhar Santhanam
}

Mechanical Engineering Department, Villanova University, Villanova, PA 19085, USA

Correspondence should be addressed to Kei-Peng Jen; kei-peng.jen@villanova.edu

Received 22 February 2013; Revised 22 April 2013; Accepted 7 May 2013

Academic Editor: Diego Gomez-Garcia

Copyright (C) 2013 Kei-Peng Jen et al. This is an open access article distributed under the Creative Commons Attribution License, which permits unrestricted use, distribution, and reproduction in any medium, provided the original work is properly cited.

\begin{abstract}
Papers of silicon nitride nanofibers were synthesized by a carbothermal reduction process. These nanofiber papers were synthesized in situ and did not require a secondary processing step. The process utilized silica nanopowders and silica gel as the precursor material. Processing geometry played a crucial role in regulating the growth of the nanofiber papers. Characterization of the nanofiber papers indicated that the nanofibers were of the alpha silicon nitride phase. Both mechanical stiffness and strength of the nanofiber papers were measured. Thermal conductivity and specific heat of the papers were also measured and were found to be lower than many common thermal insulation materials at much smaller thicknesses and were comparable to those values that are typically reported for carbon-nanotube-based buckypaper. Results of the mechanical and thermal characterization indicate that these silicon nitride nanofiber papers can be utilized for specialized thermal insulation applications.
\end{abstract}

\section{Introduction}

One-dimensional nanostructures of silicon nitride have been investigated intensively in the recent past [1-16]. These structures include nanowires, nanorods, and nanofibers. The reason for the interest in silicon nitride is because of the promise it offers in applications in optics, semiconductors, thermal insulation, and in the strengthening and toughening of materials [17].

There are several processing routes available to make these one-dimensional nanostructures of silicon nitride. Kim et al. [18] synthesized silicon nitride nanowires directly from silicon substrates. A method to fabricate silicon nitride nanorods from beta silicon carbide nanowires was developed by Bechelany et al. [15]. Carbon nanotubes were used as templates by Han et al. [19] for the synthesis of silicon nitride nanorods. Carbothermal reduction is another well-known route. Zhang et al. [11] have used it to produce silicon nitride single crystal nanowires. Arik [9] and Wu et al. [10] too have used this method. In another recent effort, Deshmukh et al. [20] fabricated silicon nitride nanofibers using carbothermal reduction with silica gel and silica nanopowders as precursors.
A recent development is the making of two-dimensional sheets or papers using one-dimensional nanostructures. Zhao et al. [21] have demonstrated a method to synthesize thin papers of carbon nanofibers. The processing conditions were controlled to produce an optimized network of carbon nanofibers. Firsich [22] discusses methods of constructing carbon nanofiber paper tailored for specific applications. Gou et al. [23] developed a carbon fiber paper for lightning strike protection in aircraft structures. Muramatsu et al. [24] produced buckypaper by filtering a stable suspension of doublewalled carbon nanotubes. Dumée et al. [25] demonstrated the use of such bucky papers as membranes for distillation. The filtration process was also used by Xu et al. [26] to make CNT paper from CNT pulp. Lu et al. [27] fabricated carbon nanofiber paper impregnated with a shape memory polymer, thereby imparting shape memory behavior to the paper.

All of the methods described so far for making twodimensional paper from one-dimensional nanostructures have relied on a second manufacturing step such as filtration to produce paper from nanofibers or nanotubes. These methods first require the production of the one-dimensional structures followed by a second method to assemble the onedimensional nanostructures into two-dimensional paper. 
Our objective in this work was to develop a process that can grow silicon nitride paper directly from the raw material precursors in a single step thus obviating the need for a twostep process. The process involved the use of carbothermal reduction. Silicon nitride nanofiber papers were grown in situ in a tube furnace on a graphite substrate. These papers were then characterized for their composition and for their mechanical and thermal properties. A description of the synthesis technique for the silicon nitride paper is provided in Section 2. Section 3 presents the results of the mechanical and thermal characterization of the fabricated silicon nitride papers. Conclusions are presented in Section 4. The results will show that the silicon nitride nanofiber papers have acceptable mechanical strength and excellent thermal insulation properties. This would make them very suitable candidates for thermal insulation applications.

\section{Synthesis Procedure for Silicon Nitride Paper}

A carbothermal reduction process was utilized to synthesize silicon nitride nanofiber paper (SNNP). The entire process consisted of three major steps. The first step involved preparation of raw pellets of the precursor material. This was followed by the construction of an interlaced stacking configuration of graphite plates, raw pellets, and graphite rings. Finally, a carbothermal reduction temperature cycle was used for the synthesis.

The precursor silica sources for the raw pellets were a mixture of silica gel (Davisil grade 643, pore size 150 Angstroms, 200-425 mesh, Sigma Aldrich) and silica nanopowders (10$20 \mathrm{~nm}$ (TEM), 99.5\% trace metals basis, Sigma Aldrich). The silica precursor powders were mixed with activated charcoal, in a mass ratio of $10: 1$, in a methanol solution. The liquid suspension was then ball milled for 15 minutes. The solution was dried at $100^{\circ} \mathrm{C}$ overnight in an oven. Next, the powder mixtures were mixed with Poly Vinyl Alcohol (PVA, Elvanol, 71-30, http://chemicalstore.com/) binder and molded into raw pellets ( $25 \mathrm{~mm}$ diameter) using a cold press. The raw pellets were dried overnight at room temperature.

After drying, the raw pellets were interlaced with graphite plates and rings and placed in a vertical cylindrical graphite crucible. In order to ensure that all surfaces of the graphite plates and the raw pellets were exposed to reacting gases in the subsequent carbothermal reduction process, the graphite rings were placed between the raw pellets and the graphite plates. The thickness of the graphite rings provided a suitable gap between the pellets and graphite plates. The dimensions of graphite plates were $31 \mathrm{~mm}$ by $31 \mathrm{~mm}$ by $3 \mathrm{~mm}$. The graphite rings to support the raw pellets had outside and inside diameters of $30 \mathrm{~mm}$ and $25.4 \mathrm{~mm}$, respectively. The thickness of the graphite rings was made slightly over $3 \mathrm{~mm}$. The vertical cylindrical graphite crucible had an outside diameter of $43.45 \mathrm{~mm}$, with a wall thickness of $6.7 \mathrm{~mm}$. A large flat graphite plate, $63 \mathrm{~mm}$ by $63 \mathrm{~mm}$, was used to support the entire cylindrical crucible and the interlaced layers. Figure 1 shows the configuration of the stacked interlaced layers.

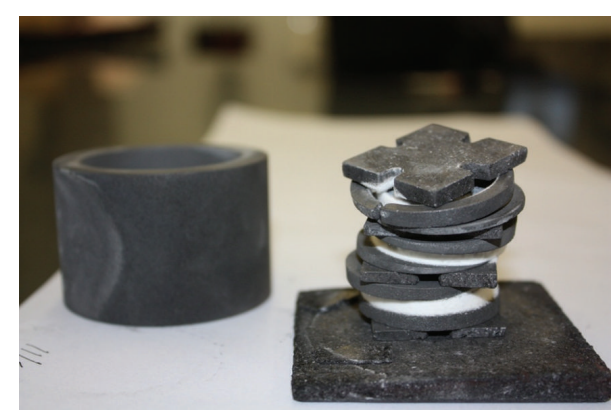

FIGURE 1: A vertical stacking arrangement of alternating raw pellets, graphite plates, and graphite rings.

The whole crucible, including the interlaced layers of graphite plates, graphite rings, and raw pellets, was then placed in a horizontal high temperature alumina tube furnace (CM Furnace Model 1730-12HT). After replacing the air with high purity $\mathrm{N}_{2}$, the graphite crucible was heated to $1450^{\circ} \mathrm{C}$ in 8 hours and kept at that temperature for another 6 hours. The furnace was cooled down to room temperature in 8 hours. Nitrogen gas was streamed through the alumina tube furnace at a rate of $20 \mathrm{sccm}$ (standard cubic centimeters per minute) during the entire heating and cooling cycle.

After the carbothermal synthesis cycle was complete, the graphite crucible assembly was removed from the furnace. Figure 2 shows a disassembled stack of pellets, graphite plates and rings, after the completion of a thermal cycle. The growth of silicon nitride fibers is evident in many of the elements of the stack. When the synthesis is done properly, the nanofibers are of a pure white color with a cotton-like appearance on the graphite plates. Silicon nitride nanofibers showed a preference for growth on the surfaces of the graphite plates rather than the raw material pellet surfaces. Layers grew to a thickness of 2.5 to $3.0 \mathrm{~mm}$ on the graphite plates. Figure 3 shows that the nanofiber layers can be easily removed in the form of intact, thin papers from the graphite plates using a razor blade. It proved difficult to extract viable nanofiber papers from the surfaces of the raw pellets because of the lack of reasonable growth on the pellets. Moreover, contamination proved to be a problem since the pellets contain loose powders that could not be separated from the nanofiber papers easily. Silicon nitride nanofiber layers were also seen on the walls of the graphite crucible and graphite rings. Clearly, graphite surfaces are a favorable place for the silicon nitride nanofiber layers/papers to grow in the carbothermal synthesis process.

\section{Characterization of Silicon Nitride Nanofiber Papers}

The silicon nitride nanofiber papers (SNNP) were characterized in many different ways. The morphology and composition of the nanofibers was established using scanning electron microscopy and X-ray diffraction. The physical density of the paper was measured. Tensile tests were conducted to estimate the modulus and strength of the silicon nitride 


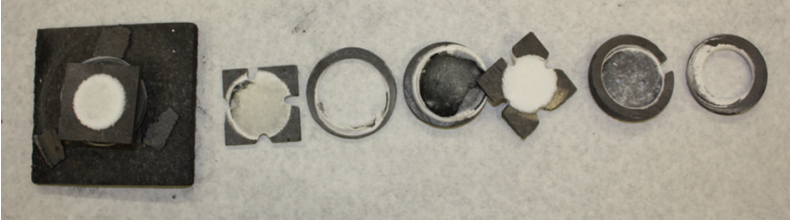

Figure 2: A disassembled stack of graphite plates, raw pellets, and graphite rings. White layers of silicon nitride nanofibers can be seen on many of the elements of the stack.

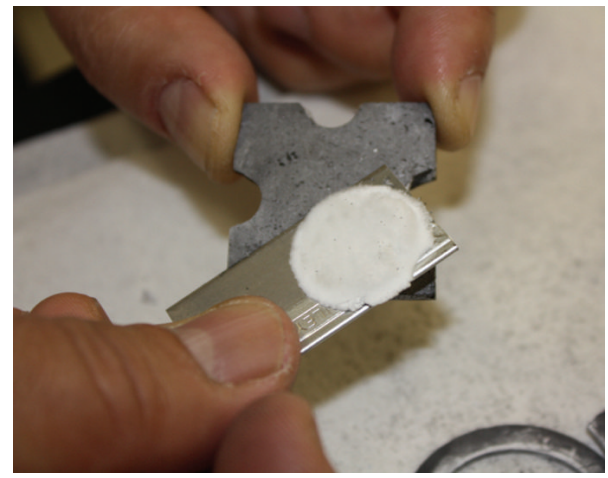

FIGURE 3: A thin silicon nitride nanofiber paper extracted from the surface of a graphite plate after the carbothermal reduction process.

paper. Thermal conductivity was measured in the paper thickness direction. The heat capacity of the paper was measured as well.

3.1. Characterization of Nanofibers in Paper. The morphology of the nanofibers was characterized using high resolution scanning electron microscopy (Hitachi S-4800). Figure 4 shows nanofibers grown on the graphite plate substrate. The fibers are several tens of microns long, with some reaching a length of 100 microns or more.

Figure 5 shows the Energy Dispersive Spectroscopy (EDS, Hitachi S-4800 with Oxford EDS) pattern from a representative sample of the nanofibers, clearly indicating strong silicon and nitrogen peaks, providing evidence for silicon nitride as the chemical composition. Figure 6 shows the ATRFTIR spectrum (Perkin Elmer Spectrum One) indicating the presence of silicon nitride.

The X-ray powder diffraction pattern (Siemens D500) in Figure 7 shows the presence of crystalline alpha silicon nitride, thus confirming that the nanofibers are crystalline alpha silicon nitride. $\mathrm{Cu} \mathrm{K}$-alpha radiation was used as the source with a step size of $0.04^{\circ}$ and dwell time of 1 second.

3.2. Density Measurement. A single sheet of silicon nitride nanofiber paper is not stiff enough to measure the physical density; therefore, three to four layers of the nanofiber papers were pressed together, with a pressure of $500 \mathrm{psi}$, to form a thicker sheet, in the shape of a disk. Then the mass and volume of the disk were measured to calculate the average physical bulk density. An average density based on five disks

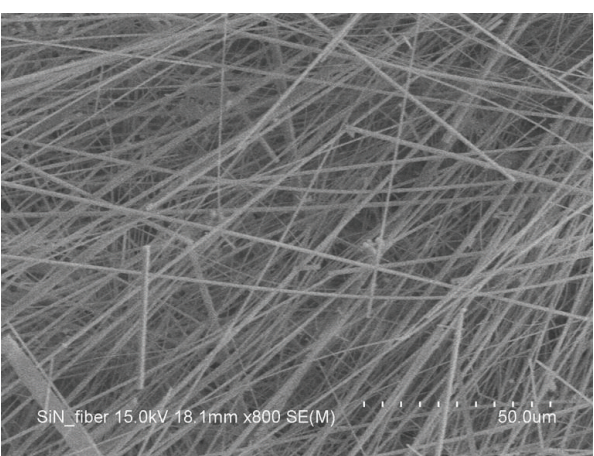

FIgURE 4: Silicon nitride nanofibers grown on a graphite plate substrate.

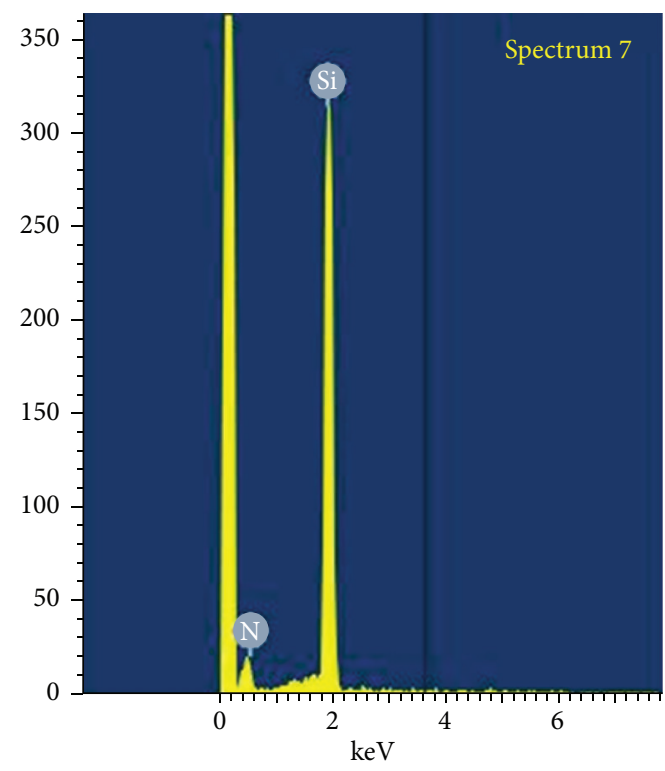

Full scale 362 cts cursor: $4.114(1 \mathrm{cts})$

FIGURE 5: Energy Dispersive Spectroscopy pattern of nanofibers indicating strong peaks of silicon and nitrogen.

was found to be $0.068 \mathrm{grams} / \mathrm{cm}^{3}$. This is somewhat lower than the densities of common cellulosic paper.

3.3. Tensile Tests. Tension tests for the nanofiber papers were conducted on an MTS Bionix universal testing machine with a crosshead speed at $25.4 \mathrm{~mm}$ per minute. The maximum capacity of the load cell was only 2 Newtons. Tensile test coupons were cut from the synthesized papers. The tensile coupons were about $20 \mathrm{~mm}$ long and $7 \mathrm{~mm}$ wide. The average thickness was about $0.15 \mathrm{~mm}$. Since strain gages cannot be mounted on such small tensile specimens, only the load versus crosshead extension data was available to estimate the peak stress and the elastic modulus. Seven tensile specimens were tested. The elastic modulus of these specimens had an average value of $503 \mathrm{kPa}$ with a standard deviation of $181 \mathrm{kPa}$. The peak stress (strength) had an average value of $50.9 \mathrm{kPa}$ with a standard deviation of $15.2 \mathrm{kPa}$. This strength can be compared with that of typical bond (cellulosic) paper, which 


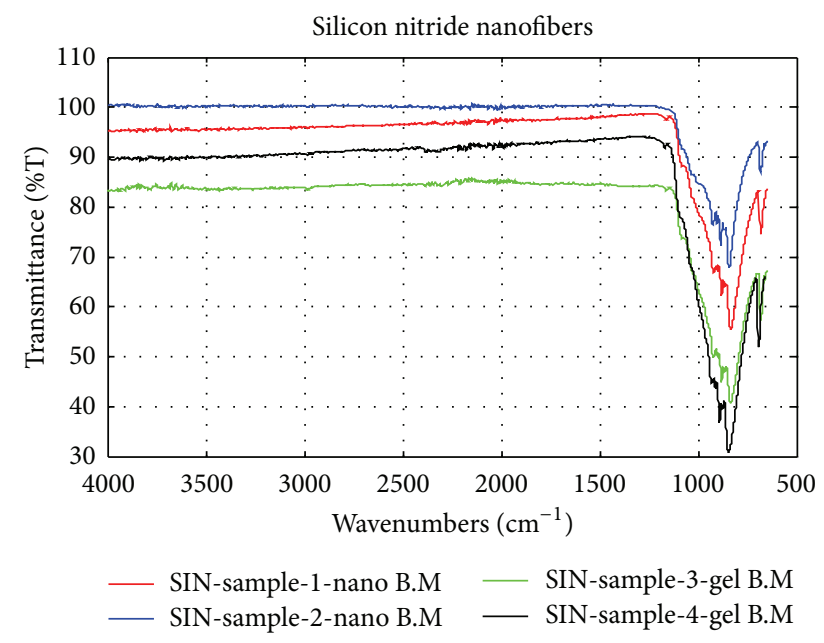

FIGURE 6: ATR-FTIR spectra of nanofibers indicating strong absorption corresponding to silicon nitride.

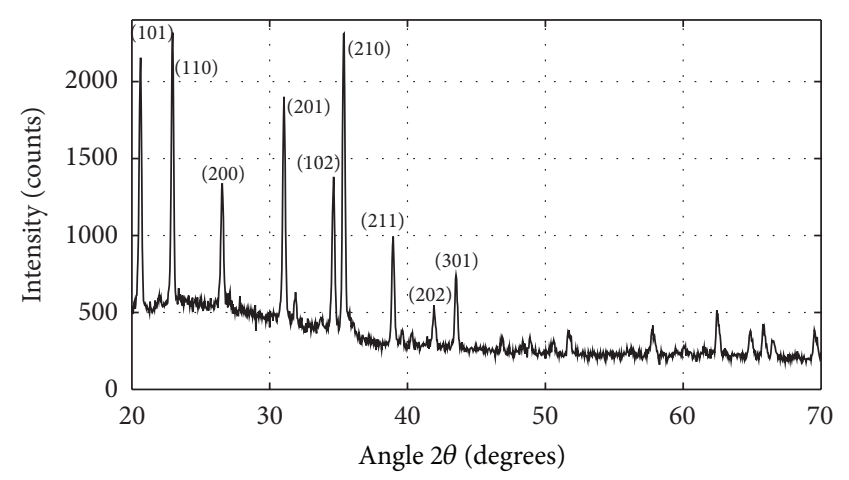

FIgURE 7: X-ray diffraction pattern for the nanofibers indicating alpha silicon nitride.

has a strength in the range of $250-300 \mathrm{kPa}$. Obviously the strength of the silicon nitride nanofiber papers is only about $1 / 5$ of typical bond office paper. However, Figure 8 indicates that the silicon nitride nanofiber papers do have entangled strength among the nanofibers, so that the paper has the typical tensile stress versus crosshead extension curve seen in many common materials.

\subsection{Thermal Conductivity Measurements of Silicon Nitride} Paper. In this study, a cut-reference bar apparatus was used to determine the effective thermal conductivity of silicon nitride nanofiber papers according to ASTM Standard D5470 [28]. Briefly, the cut-reference bar apparatus (shown in Figure 9) uses a set of two constant temperature plates to direct heat through two independent, oxygen-free high conductivity (OFHC) copper reference bars $\left(k_{\mathrm{OFHC}}=391 \mathrm{~W} / \mathrm{m} / \mathrm{K}\right)$, in the middle of which rests a sample with known thickness and unknown thermal conductivity. Each constant temperature plate contains a three-pass flow loop for even heat spreading to the copper reference bars. The fluid passed through each plate is thermally controlled via constant temperature bath. Externally, an insulating material (fiberglass insulation,

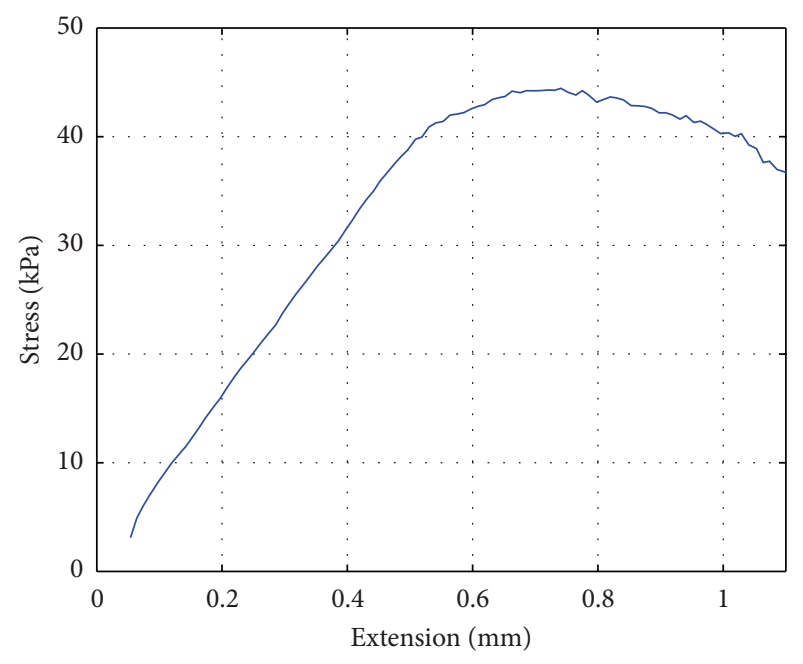

FIgURE 8: Tensile stress versus extension for a silicon nitride nanofiber paper.

$k_{\text {insulation }}=0.14 \mathrm{~W} / \mathrm{m} / \mathrm{K}$ ) is used to cover the reference bars in order to achieve one-dimensional thermal transport across the material of interest. The insulating material also doubles as a method to minimize convective and radiative heat losses from the sides of the reference bars. The effective thermal conductivity is calculated from data obtained by a series of thermocouples embedded within the reference bars. The thermocouples are calibrated using a constant temperature bath and a NIST calibrated thermometer over a temperature range of $0^{\circ} \mathrm{C}$ to $110^{\circ} \mathrm{C}$. Each set of thermocouples is inserted into the center of each reference bar and each individual thermocouple is spaced $12.7 \mathrm{~mm}$ apart along the length of each reference bar in order to obtain a linear temperature gradient from source to sink. In this study, the heat flow is allowed to reach a steady-state condition over a period of 30 minutes before the thermocouple temperatures are read and used for calculation. Additionally, a piston is located below the lower cold plate in order to keep the material at a constant pressure for the duration of testing.

For this work, the thermal impedance, $\theta$, of the silicon nitride nanofiber papers of thickness $t$ is calculated using

$$
\theta=\frac{A_{\text {sample }}}{Q} \cdot \Delta T .
$$

In (1), $Q$ represents the average heat flow through the top and bottom reference bars of Figure 9. $A_{\text {sample }}$ is the crosssectional area of the silicon nitride paper sample. $\Delta T$ is the temperature difference between the top and bottom of the silicon nitride paper sample and is determined using a linear interpolation process.

The thermal impedance across three different silicon nitride paper samples, each with different thickness, was determined experimentally. Subsequently, the thermal impedance was plotted as a function of the material's thickness (Figure 10), and the effective thermal conductivity of the material was then obtained via linear regression through the thermal impedance data. The reciprocal of the slope of the 


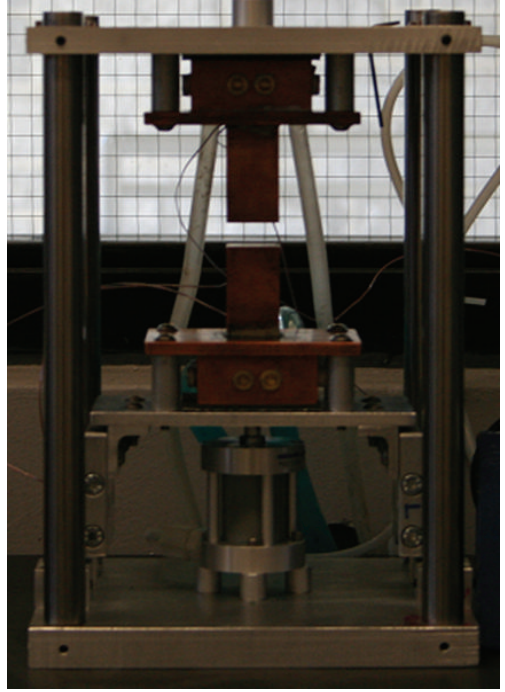

Figure 9: Cut-reference bar apparatus used for the measurement of the thermal conductivity of silicon nitride papers.

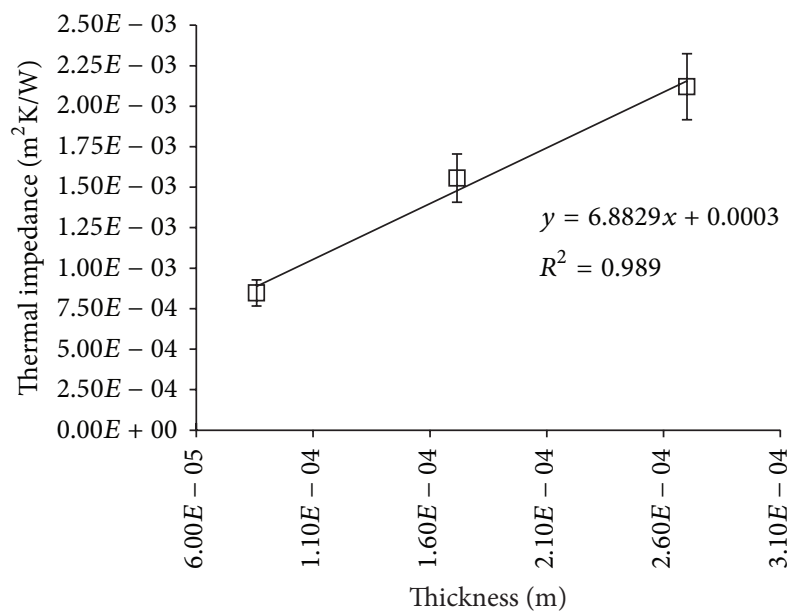

FIGURE 10: Thermal impedance versus sample thickness for silicon nitride nanofiber paper.

linear regression represents the effective thermal conductivity of the silicon nitride paper as shown in (2). The associated uncertainty of (2) is found to be $\pm 9.6 \%$ using a standard estimation of uncertainty, which can be found in [29],

$$
\lambda=\frac{1}{6.8829} \cdot \frac{m}{\left(\left(K \cdot m^{2}\right) / W\right)}=0.14 \frac{W}{m \cdot K} .
$$

From these results, it is clear that the silicon nitride nanofiber paper behaves as a thermal insulator. This result is in stark contrast to the reported thermal conductivity of individual silicon nanostructures and bulk silicon nitride, which have been shown to be moderate to exceptional thermal conductors (between 3.2 and $100 \mathrm{~W} / \mathrm{m} / \mathrm{K}$ ) and is comparable to that which has been found for carbon nanotube-based buckypaper [30]. Thus, this silicon nitride nanofiber paper offers great promise for thermal insulation applications.
3.5. Measurement of the Specific Heat Capacity of Silicon Nitride Nanofiber Paper. The heat capacity of silicon nitride nanofiber papers was measured using a TA Q200 Differential Scanning Calorimeter (DSC) in accordance with ASTM E1269 [31]. In order to calculate the heat capacity, the energy absorbed (referred to as the "heat value") by an empty sample pan was measured as a function of temperature and compared to a reference sample (i.e. sapphire). The difference between the measured values can be used to quantify the specific heat of an unknown sample as a function of temperature. In accordance with ASTM E1269, the heat rate during operation was set to $20^{\circ} \mathrm{C} / \mathrm{min}$ and a nitrogen purge gas was supplied into the sample chamber at a flow rate of $50 \mathrm{~mL} / \mathrm{min}$ in order to establish an adiabatic boundary condition at the sample pans' edges. The three heat flow values (empty pan, sapphire, and the sample) were then used to compute the specific heat of the sample, which was calculated according to

$$
\begin{gathered}
c_{p}(T)=\frac{60 \cdot E(T) D_{s}(T)}{W_{s} \cdot b}, \\
E(T)=\left[\frac{b}{\left(60 \cdot D_{s t}(T)\right)}\right] \cdot\left[W_{s t} \cdot c_{p s t}(T)\right] .
\end{gathered}
$$

In (3), $c_{p}$ represents specific heat capacity $(\mathrm{J} / \mathrm{kg} / \mathrm{K}), E(T)$ is the calorimetric sensitivity (defined by (4)), $D_{s}$ is the difference between the DSC heat values for the unknown sample and the sapphire sample $(\mathrm{mW}), W_{s}$ is the mass of the unknown sample $(\mathrm{mg})$, and $b$ is the heating rate $\left({ }^{\circ} \mathrm{C} / \mathrm{min}\right)$. In (4), $D_{s t}$ represents the difference between the DSC heat values for the sapphire sample and the empty reference pan $(\mathrm{mW}), W_{s t}$ is the mass of the sapphire reference material $(\mathrm{mg})$, and $c_{p s t}$ is the specific heat capacity of the sapphire reference material as a function of temperature $(\mathrm{J} / \mathrm{kg} / \mathrm{K})$.

The temperature dependent heat values of the empty reference pan, the sapphire reference material, and the silicon nitride nanofiber paper samples are shown in Figure 11.

The calculated specific heat values of the silicon nitride paper samples (using (3) and (4)) are shown in Figure 12 as a function of temperature of the samples. For this study, the specific heat capacity of the silicon nitride nanofiber paper samples was determined for temperatures ranging between $35^{\circ} \mathrm{C}$ and $80^{\circ} \mathrm{C}$ at intervals of $5^{\circ} \mathrm{C}$.

As can be seen in Figure 12, the specific heat capacity does not deviate significantly from its mean value (average $C_{p}$ $=0.8 \mathrm{~J} / \mathrm{g} / \mathrm{K}$, standard deviation $=0.01 \mathrm{~J} / \mathrm{g} / \mathrm{K}$ ) as temperature increases. The uncertainty of this method was found to be $1.9 \%$ according to [29]. The relatively low specific heat of the silicon nitride nanofiber paper indicates that it takes longer for its temperature to increase to a steady-state condition, or desired temperature, compared with bulk plasma enhanced chemical vapor deposition (PECVD) silicon nitride, which has a specific heat capacity of $0.17 \mathrm{~J} / \mathrm{g} / \mathrm{K}$. The silicon nitride nanofiber paper also exhibits a specific heat capacity that differs from other bulk nanostructure papers, such as carbon nanotube (CNT) papers, which have specific heat capacities on the order of $0.1 \mathrm{~J} / \mathrm{g} / \mathrm{K}$, making silicon nitride nanofiber papers a more desirable choice for use as thermally insulating nanomaterials in cyclically operating energy systems. 


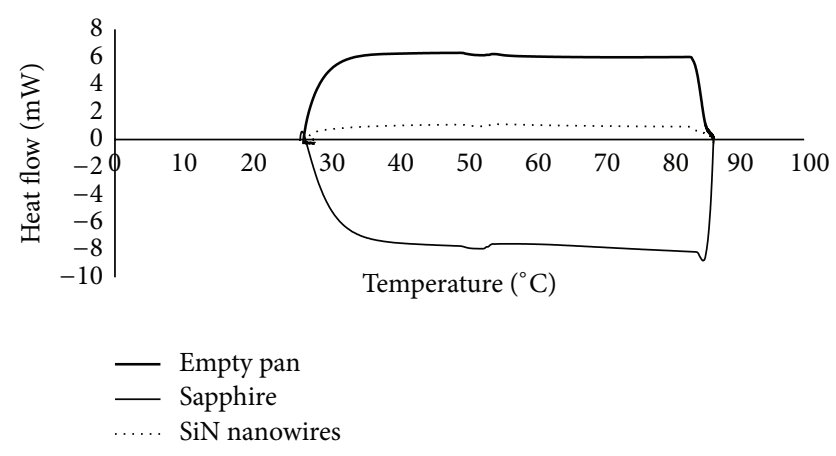

Figure 11: Heat flow values used to calculate the specific heat of a silicon nitride nanofiber paper sample.

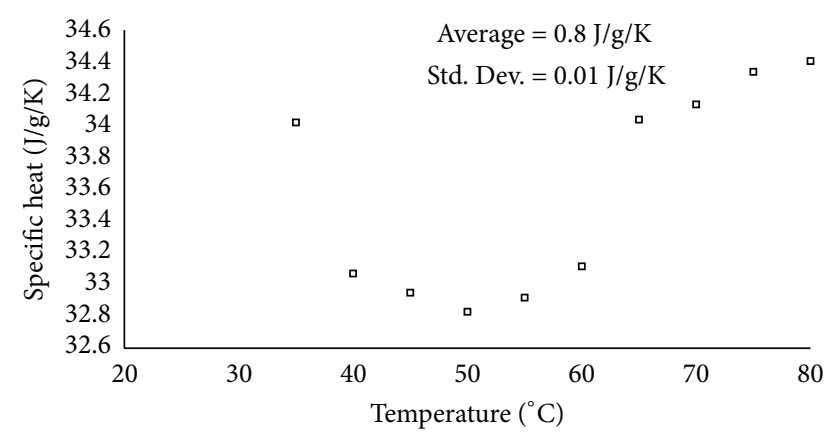

FIGURE 12: Specific heat of silicon nitride nanofiber paper as a function of temperature.

\section{Conclusions}

Crystalline silicon nitride nanofiber papers were formed in situ on the surface of graphite plates by the carbothermal reduction method using silica nanopowder and silica gel precursor pellets. The carbothermal reduction process was performed at a temperature of $1450^{\circ} \mathrm{C}$ in a flowing nitrogen atmosphere inside a horizontal alumina tube furnace. A special stacking arrangement of graphite substrate plates and silica nanopowder pellets was utilized to ensure adequate exposure of the substrate surfaces to the reacting gases. This arrangement allowed multiple substrate surfaces to be exposed, thereby allowing the growth of the nanofiber papers on multiple surfaces, thus increasing the yield. Nanofiber papers can be easily extracted from the graphite plates with very low contamination. The silicon nitride nanofiber papers were characterized both mechanically and thermally. The average mechanical strength of in situ synthesized paper was $50.9 \mathrm{kPa}$ with an average modulus of $503 \mathrm{kPa}$. The thermal conductivity of the paper samples was measured at $0.14 \mathrm{~W} / \mathrm{mK}$, while the specific heat capacity was determined to be $0.8 \mathrm{~J} / \mathrm{g} / \mathrm{K}$. These measurements indicate that the silicon nitride nanofiber papers, synthesized in situ by the carbothermal process, offer plenty of promise for use as thermal insulation materials in specialized applications.

\section{References}

[1] G. Li, X. Li, H. Wang, and Z. Li, "Long silicon nitride nanowires synthesized in a simple route," Applied Physics A, vol. 93, no. 2, pp. 471-475, 2008.

[2] F. Wang, G. Jin, and X. Guo, "Formation mechanism of $\mathrm{Si}_{3} \mathrm{~N}_{4}$ nanowires via carbothermal reduction of carbonaceous silica xerogels," Journal of Physical Chemistry B, vol. 110, no. 30, pp. 14546-14549, 2006.

[3] D. Pan, Z. Shuyuan, Y. Chen, and J. G. Hou, "Hydrothermal preparation of long nanowires of vanadium oxide," Journal of Materials Research, vol. 17, no. 8, pp. 1981-1984, 2002.

[4] C. N. R. Rao, G. Gundiah, F. L. Deepak, A. Govindaraj, and A. K. Cheetham, "Carbon-assisted synthesis of inorganic nanowires," Journal of Materials Chemistry, vol. 14, no. 4, pp. 440-450, 2004.

[5] X. C. Wu, W. H. Song, B. Zhao et al., "Synthesis of coaxial nanowires of silicon nitride sheathed with silicon and silicon oxide," Solid State Communications, vol. 115, no. 12, pp. 683-686, 2000.

[6] C. Deng, P. Yu, M. Yau, C. Ku, and D. H. L. Ng, "Fabrication of single-crystal $\alpha-\mathrm{Al}_{2} \mathrm{O}_{3}$ nanorods by displacement reaction," Journal of the American Ceramic Society, vol. 86, no. 8, pp. 13851388, 2003.

[7] W. Yang, Z. Xie, J. Li, H. Miao, L. Zhang, and L. An, "Ultra-long single-crystalline $\alpha$-Si3N4 nanowires: derived from a polymeric precursor," Journal of the American Ceramic Society, vol. 88, no. 6, pp. 1647-1650, 2005.

[8] Y. Zhang, N. Wang, R. He, J. Liu, X. Zhang, and J. Zhu, "A simple method to synthesize $\mathrm{Si}_{3} \mathrm{~N}_{4}$ and $\mathrm{SiO}_{2}$ nanowires from $\mathrm{Si}$ or $\mathrm{Si} / \mathrm{SiO}_{2}$ mixture," Journal of Crystal Growth, vol. 233, no. 4, pp. 803-808, 2001.

[9] H. Arik, "Synthesis of $\mathrm{Si}_{3} \mathrm{~N}_{4}$ by the carbo-thermal reduction and nitridation of diatomite," Journal of the European Ceramic Society, vol. 23, no. 12, pp. 2005-2014, 2003.

[10] X. C. Wu, W. H. Song, W. D. Huang et al., "Simultaneous Growth of alpha silicon nitride and beta silicon carbide nanorods," Materials Research Bulletin, vol. 36, pp. 847-852, 2001.

[11] L. D. Zhang, G. W. Meng, and F. Phillipp, "Synthesis and characterization of nanowires and nanocables," Materials Science and Engineering A, vol. 286, no. 1, pp. 34-38, 2000.

[12] G. M. Chaudhuri, S. K. Ahmadullah, R. Dey, G. C. Das, S. Mukherjee, and M. K. Mitra, "Novel technique for synthesis of silicon nitride nanowires," Advances in Applied Ceramics, vol. 110, no. 4, pp. 211-214, 2011.

[13] G. J. Qi, "Si-N-C nanowires derived by polyhydridomethylsilazane pyrolysis," Materials Sciences and Applications, vol. 2, pp. 936-939, 2011.

[14] C. Xu, M. Kim, J. Chun et al., "Gallium-doped silicon nitride nanowires sheathed with amorphous silicon oxynitride," Scripta Materialia, vol. 53, no. 8, pp. 949-954, 2005.

[15] M. Bechelany, A. Brioude, S. Bernard, G. Ferro, D. Cornu, and P. Miele, "Large-scale preparation of faceted $\mathrm{Si}_{3} \mathrm{~N}_{4}$ nanorods from $\beta$-SiC nanowires," Nanotechnology, vol. 18, no. 33, Article ID 335305, pp. 1-6, 2007.

[16] S. Motojima, S. Ueno, T. Hattori, and K. Goto, "Growth of regularly coiled spring-like fibers of $\mathrm{Si}_{3} \mathrm{~N}_{4}$ by iron impurityactivated chemical vapor deposition," Applied Physics Letters, vol. 54, no. 11, pp. 1001-1003, 1989.

[17] J. J. Melendez-Martinez and A. Dominguez-Rodriguez, "Creep of silicon nitride," Progress in Materials Science, vol. 49, no. 1, pp. 19-107, 2004. 
[18] H. Y. Kim, J. Park, and H. Yang, "Synthesis of silicon nitride nanowires directly from the silicon substrates," Chemical Physics Letters, vol. 372, no. 1-2, pp. 269-274, 2003.

[19] W. Han, S. Fan, Q. Li, B. Gu, X. Zhang, and D. Yu, "Synthesis of silicon nitride nanorods using carbon nanotube as a template," Applied Physics Letters, vol. 71, no. 16, pp. 2271-2273, 1997.

[20] S. Deshmukh, K. P. Jen, and S. Santhanam, "Comparison of silicon nitride nanofibers synthesized using silica nanopowders and silica gel," Materials Sciences and Applications, vol. 3, pp. 523-529, 2012.

[21] Z. Zhao, J. Gou, and A. Khan, "Processing and structure of carbon nanofiber paper," Journal of Nanomaterials, vol. 2009, Article ID 325769, 7 pages, 2009.

[22] D. W. Firsich, "Varieties of carbon nanofiber paper," SAMPE Journal, vol. 43, no. 4, pp. 58-61, 2007.

[23] J. Gou, Y. Tang, F. Liang, Z. Zhao, D. Firsich, and J. Fielding, "Carbon nanofiber paper for lightning strike protection of composite materials," Composites B, vol. 41, no. 2, pp. 192-198, 2010.

[24] H. Muramatsu, T. Hayashi, Y. A. Kim et al., "Pore structure and oxidation stability of double-walled carbon nanotube-derived bucky paper," Chemical Physics Letters, vol. 414, no. 4-6, pp. 444-448, 2005.

[25] L. F. Dumée, K. Sears, J. Schütz et al., "Characterization and evaluation of carbon nanotube Bucky-Paper membranes for direct contact membrane distillation," Journal of Membrane Science, vol. 351, no. 1-2, pp. 36-43, 2010.

[26] G. Xu, Q. Zhang, W. Zhou, J. Huang, and F. Wei, “The feasibility of producing MWCNT paper and strong MWCNT film from VACNT array," Applied Physics A, vol. 92, no. 3, pp. 531-539, 2008.

[27] H. Lu, Y. Liu, J. Gou, J. Leng, and S. Du, "Electrical properties and shape-memory behavior of self-assembled carbon nanofiber nanopaper incorporated with shape-memory polymer," Smart Materials and Structures, vol. 19, pp. 1-7, 2010.

[28] ASTM International, "Standard test method for thermal transmission properties of thermally conductive electrical insulation materials," ASTM D5470-06.

[29] R. J. Warzoha, A. Rao, R. Weigand, and A. S. Fleischer, "Experimental characterization of the thermal diffusivity of paraffin phase change material embedded with herringbone style graphite nanofibers," in Proceedings of the ASME Summer Heat Transfer Conference (SHTC '12), Rio Grande, Puerto Rico, USA, July 2012.

[30] R. S. Prasher, X. J. Hu, Y. Chalopin et al., "Turning carbon nanotubes from exceptional heat conductors into insulators," Physical Review Letters, vol. 102, no. 10, Article ID 105901, 4 pages, 2009.

[31] ASTM International, "Standard test method for determining specific heat capacity by differential scanning calorimetry," ASTM E1269-11. 

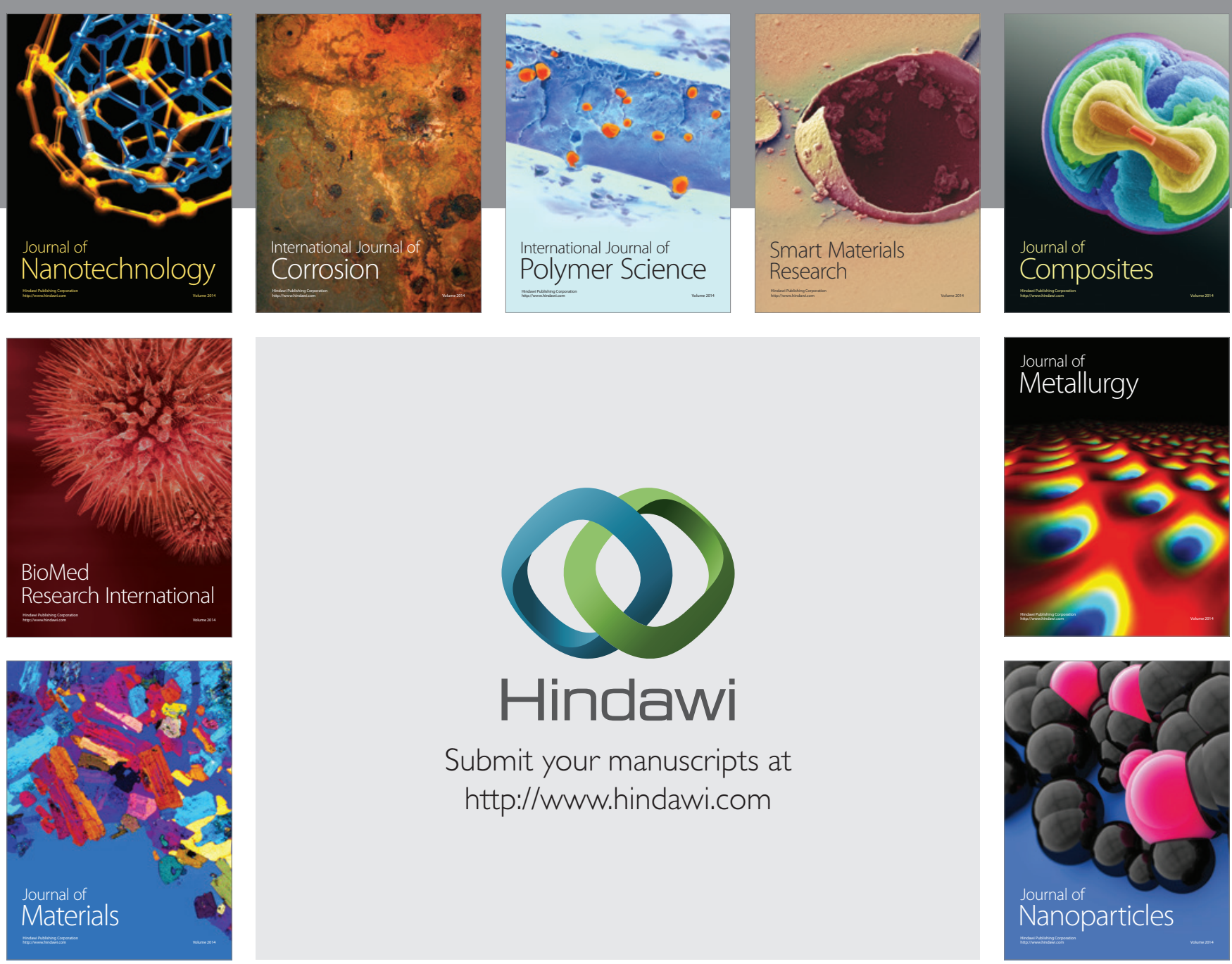

Submit your manuscripts at http://www.hindawi.com
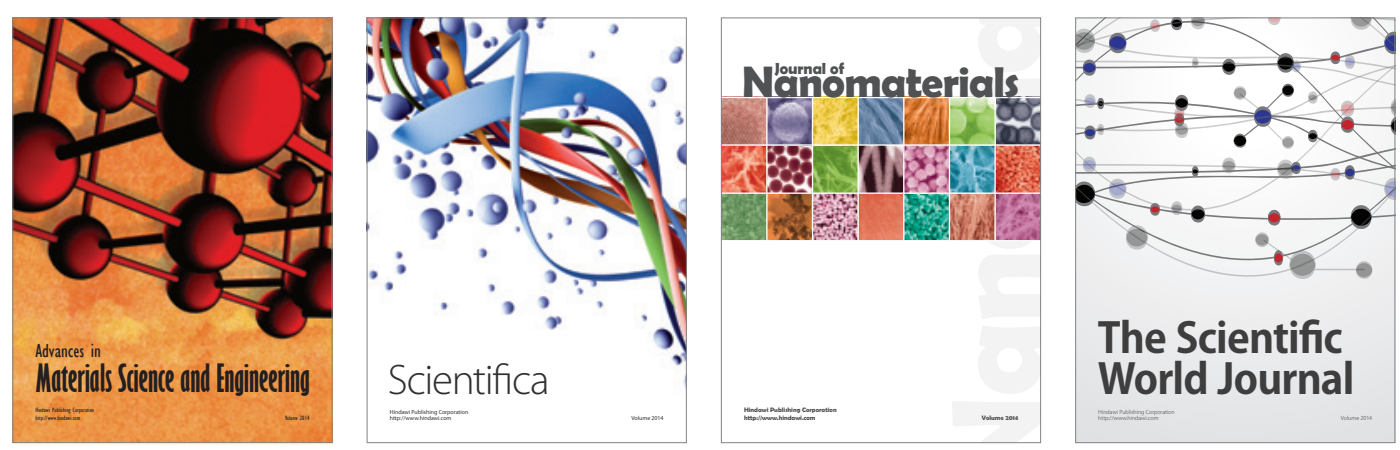

\section{The Scientific World Journal}
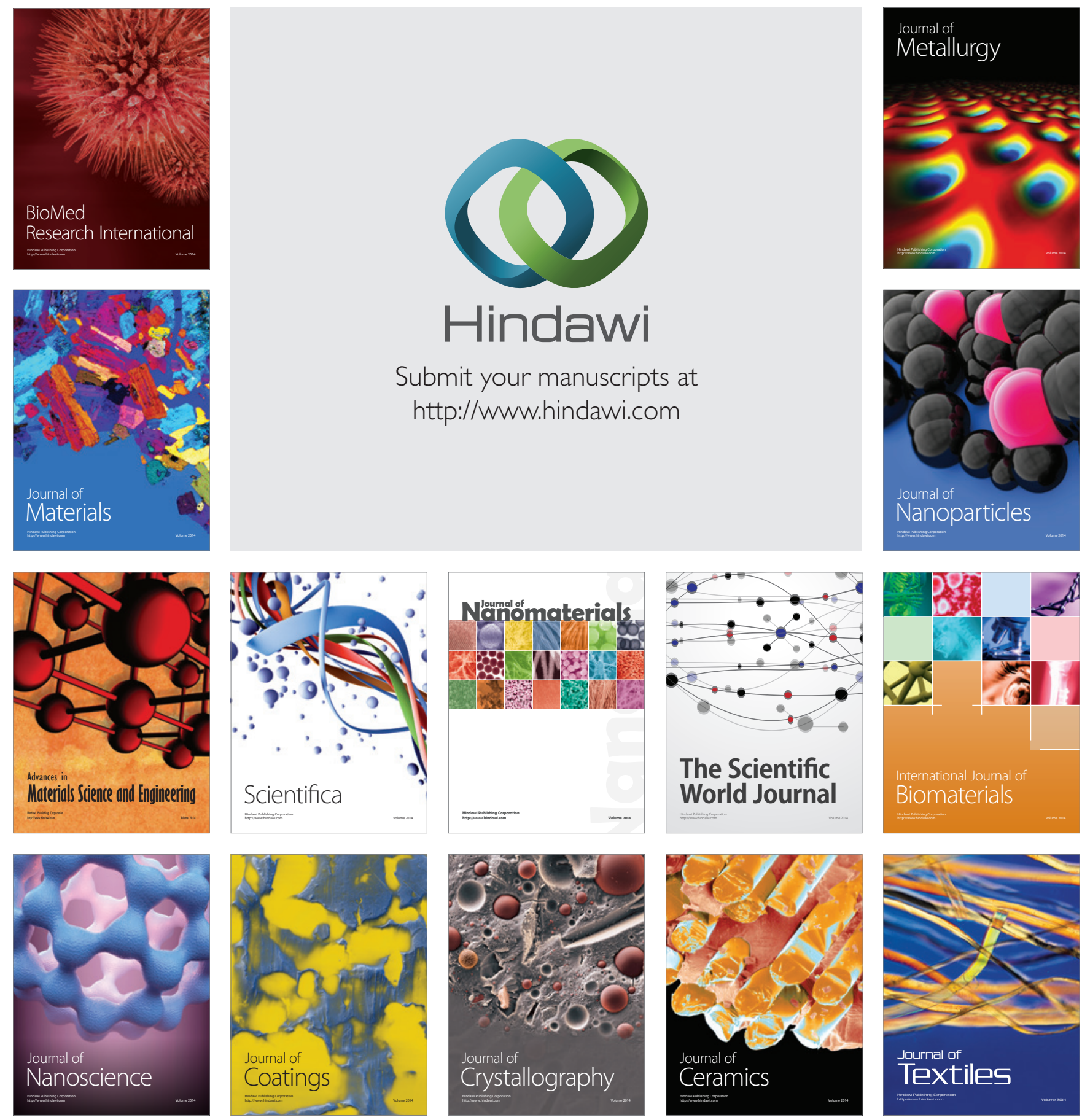